\title{
Multiple mechanisms of small RNAs
}

Different types of non-coding RNAs have diverse roles in gene regulation. A new study shows how a single quorum regulatory RNA (Qrr) can regulate target RNAs by distinct mechanisms during bacterial quorum sensing.

Vibrio species of bacteria use multiple related Qrr RNAs, each $\sim 100$ nucleotides in length, in a quorum-sensing network that controls gene expression in response to cell population density. To study the mechanisms by which one of the Qrr family members, Qrr3, regulates different RNA targets, Feng et al. engineered a competition assay in Escherichia coli involving inducible Qrr3 RNA and two inducible target mRNAs in which different coloured fluorescent reporter proteins are encoded downstream of Qrr3-binding sites in the target mRNAs. The fates of the Qrr3 RNA and target mRNAs were tracked by Northern blotting, and expression of the encoded fluorescent proteins was monitored by flow cytometry.

The investigators identified distinct modes of action of Qrr3 on different target mRNAs: nucleolytic degradation of the target mRNA (with or without simultaneous degradation of Qrr3), sequestration of the target mRNA, and activation of translation from the target mRNA with concomitant Qrr3 degradation.

Previous experimental work and RNA-RNA binding predictions showed that all of these regulatory modes involve the hybridization of Qrr3 to its targets, so Feng et al. generated mutant forms of the Qrr-binding sites in the target mRNAs to test what particular features of base-pairing determine the regulatory mode. For example, they showed that when hybridization to the target RNA disrupts a 5 ' hairpin structure in Qrr3, this leaves Qrr3 susceptible to degradation, and that the overall binding strength of the Qrr3-target interaction determines the degree of Qrr3 sequestration.

The authors used computational modelling to show that the different regulatory modes have distinct potencies and dynamics in regulation of target mRNAs. Moreover, they validated that different target mRNAs showed the predicted dynamics of regulation in Vibrio harveyi strains. As confirmation that these different modes are functionally relevant, altering the regulatory mode by replacing the Qrr3-binding site in the luxR target mRNA with that from luxM or luxO disrupted quorum-sensing dynamics.

Overall, this study highlights how multiple regulatory mechanisms can be embedded in small RNAs to confer precise regulatory dynamics. Further work could determine how prevalent these combined mechanisms are in other systems and whether they can be adopted in designed synthetic RNAs.

Darren J. Burgess

ORIGINAL RESEARCH PAPER Feng, L. et al. A Qrr noncoding RNA deploys four different regulatory mechanisms to optimize quorum-sensing dynamics. Cell http://dx.doi.org/10.1016/ j.cell.2014.11.051 (2015)

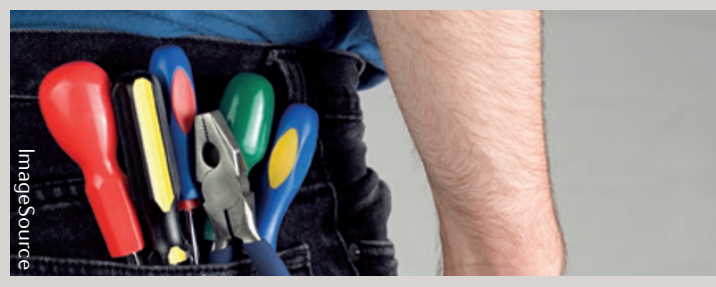

